\title{
The future synthesis of medical systems
}

\section{Editorial}

"Progress has not followed a straight ascending line, but a spiral with rhythms of progress and retrogression, of evolution and dissolution." Johann Wolfgang von Goethe (1749-1832). "If the $15^{\text {th }}$ century (medieval) was the age of Belief, the $16^{\text {th }}$ century (Renaissance) the age of Adventure, the $17^{\text {th }}$ century the age of Reason, the $18^{\text {th }}$ century the age of Enlightenment, the $19^{\text {th }}$ century the age of Ideology and the $20^{\text {th }}$ century the age of Analysis, then naturally the $21^{\text {st }}$ century should be the age of synthesis". George Christakos. Jen-Feng Wang and Jiaping Wu in 'Stochastic Medical Reasoning and Environmental Health Exposure.

\section{Imperial college press 20 | 4}

Modern scientific medicine has brought enormous benefit to humankind, antibiotics, vaccinations, surgery, and pharmacology. Some like chemotherapy and radiotherapy are effective but toxic just as other pharmaceutics manage a thin line between cure and suffering. When examined, medicine's progress is most in the past, antibiotics are 70years old, polio vaccines are 60 years old and the first information on smallpox inoculation was written in 1759, while Edward Jenner began inoculation with cowpox serum in 1770 . Modern scientific surgery began during the Age of Enlightenment in Europe. An important figures in this regard was the Scottish surgical scientist, John Hunter (1723-1798), generally regarded as the father of modern scientific surgery.

The medicine of the future will be different from the today's medicine. It will not be about breakthroughs, amazing new drugs and devises or the promise of a universal panacea. These are actually the unfulfilled promises of medicine today. Despite all the hype and great expectations, medicine has stalled it has peaked. New drug development is declining, an insight into the causes of disease is a trickle of what has gone before, there is increasing drug-resistance and most importantly medicine is increasingly inefficient in spite of an ever-increasing cost.

In the journal Nature Reviews Drug Discovery (2012) Jack W. Scannell declared "...the number of new drugs approved per billion US dollars spent on R\&D has halved roughly every 9 years since 1950 , falling around 80 -fold in inflation-adjusted terms. The last several decades have seen a relative decrease in the introduction of new drugs despite increased investment in research and development". It is important to remember pharmaceutical companies spend more on marketing than they do on research. If you look at the $R \& D$ percentages versus those for sales, general and administrative expenses (SG\&A), it's apparent that drug-makers allocate more of their budgets to SG\&A than to R\&D.

Brad Spellberg wrote in the journal Clinical Infectious Diseases (2008) that the "pharmaceutical industry is experiencing decreased productivity, pathogens that cause common diseases such as tuberculosis, pneumonia, and skin infections are increasingly developing resistance to currently available antibiotics". In 2008, the Infectious Diseases Society of America (IDSA), a leading national organization that represents infectious disease specialists, issued a
Volume I Issue 4 - 2015

\author{
Daniel Weber ${ }^{1,2}$ \\ 'Charles Sturt University, Australia \\ ${ }^{2}$ Visiting Professor Tianjin University, China
}

Correspondence: Daniel Weber, Consultant at National Cancer Institute, 74 Brandling St, Alexandria NSW 2015, Australia,Tel +61 2 95192233, Email drdweber@panaxea.com

Received: August 25, 2015 | Published: August 26, 2015

call to action to address the growing public health threats posed by antimicrobial resistance.

JA Johnson states in the Archives of Internal Medicine (1995) "Drug-related morbidity and mortality has been found to cost more than \$76billion per year in the United States. The largest component of this total was the cost of drug-related hospitalisations. Adverse drug reactions cost every man, woman, and child in the United States nearly $\$ 300$ per year".

American health care is the most expensive in the world, with 17.7 per cent of gross domestic product (GDP) spent on healthcare. This is significantly more than other developed countries. Australia, for comparison, which spends the least, spends only 8.9 per cent.

For the costs involved, you might assume Americans enjoy the best health care in the world, but this would be a mistaken assumption. According to a new report released by the Commonwealth Fund, the US ranks last or near last in all aspects of health care, from access and equity to efficiency. A review of US healthcare expenses by the Institutes of Medicine (IOM) revealed that 30 cents of every dollar spent on medical care is wasted, adding up to \$750billion annually.

Researchers have long documented that the most educated Americans were making the biggest gains in life expectancy, but now they say mortality data show that life spans for some of the least educated Americans are actually contracting.

According to the World Health Organisation (WHO), the U.S. lags far behind Canada, Australia and swaths of Western Europe in life expectancy and rivals third world countries. WHO has reported that the U.S., factoring in both genders, has an overall life expectancy of 79 ( 76 for males, 81 for females) compared to 83 in Japan and Switzerland, 82 in France, Iceland, Spain, Italy, Australia, Canada, Israel, Luxembourg, Singapore and Sweden, 81 in Germany, the Netherlands, Austria, New Zealand, Finland, South Korea, the Republic of Ireland and Norway, and 80 in Belgium, Slovenia, the U.K., Malta, Kuwait and Portugal.

Even though the United States doesn't have the highest life expectancy among the OECD nations, it does have the highest share of health spending. Health costs made up 17.7 per cent of the U.S. GDP in 2011, compared with the OECD average of 9.3 per cent. And the U.S. spent $\$ 8,508$ per capita on health that year, compared with $\$ 3,339$ on average for all OECD nations. 
A paper published in the New England Journal of Medicine predicts a decrease in life expectancy, which rose slowly but steadily last millennium. Experts say the drop will occur when the current generation of obese and overweight adults reaches old age, and will worsen when obese and overweight children hit middle age. Life expectancy in the US, about 80 years for women and 75 for men, would continue to rise for the next five to 10 years but level off and decline as more obese children reached adulthood.

Obesity has been shown to reduce the length of life by about five to 20 years. About 68 per cent of Australian men and 52 per cent of Australian women are overweight or obese, which puts them at an elevated risk of Type 2 diabetes, heart disease and cancer.

While all these are of great concern it is not fair to condemn medical science for all these failings. There are historical breakthroughs that have enhanced health and well-being but a new paradigm of medicine is needed. The future of medicine will incorporate not only the best of current medical science but also the rich history of ancient and natural medicines. A synthesis to see the future of medicine one needs to understand the history of medicine, how it developed over time. Humans are the only animals that consciously and intentionally use medicine. Sure, some animals instinctively seek grasses and barks from plants to heal themselves but it is not intentional and is forgotten when health returns. It is not passed on to future generations.

\section{Primitive medicine}

Early human medicine going back hundreds of millennia ago was mostly trial and error experimentation but early hunter-gatherer tribes developed myths around the use of plants, which were passed along to the next generation. As the use of plants expanded, medicine took on a cosmology as to why these plants worked but much of this was based on metaphysical or mystical thinking. It was not rational or logical. Superstition and medicine were closely aligned and it was called shamanism. A person got a disease because of a curse of the gods, an evil eye or an evil wind. The shaman or priest would cast spells, rattle drums as well as provide potions to drink.

Medicine also developed regionally, based upon the plants available in that area and the tribal cosmological and spirit beliefs. No one knew what worked, if indeed it did work but over time certain activities were found to be effective. Shamanism and superstition was the basis for early treatments and there was little separation between ritual, magical invocations to the gods and the use of plants to treat disease. This is the earliest stage of medicine and today is called folk medicine.

Folk medicine comprises knowledge systems that developed over generations within various societies before the era of modern medicine. The World Health Organisation (WHO) defines traditional medicine as "the sum total of the knowledge, skills, and practices based on the theories, beliefs, and experiences indigenous to different cultures, whether explicable or not, used in the maintenance of health as well as in the prevention, diagnosis, improvement or treatment of physical and mental illness".

\section{Limits to primitive medicine}

There is no distinction between medical efficacy and subjective belief, medicine is not a distinct discipline but controlled by priests or shaman caste and pathology is deemed non-physical. All medicine was regional, tribal based.

\section{Empirical medicine}

The second stage of medical evolution occurred around 2,000years ago. In China Zhang Zhongjing wrote the Shanghan Lun or Treatise on Cold Diseases, in which disease was rationally laid out in regard to cause. Zhang Zhongjing was a Han Dynasty physician and one of the most eminent Chinese physicians during the later years of the Han Dynasty. He established medication principles and summed up the medicinal experience up until that time, thus making a great contribution to the development of Traditional Chinese Medicine. In the West, Hippocrates laid the foundation of Greek Medicine, while Claudius Galenus, also called Galen further developed its theory and practice, and carried Greco-Roman medicine to its zenith. Galen's chief contributions to the theory of Greek Medicine were his theories of the three varieties of pneuma, or vital energy, and the Four Faculties of the organism. He also developed and expanded the humeral physiology and pathology of Hippocrates.

The Shanghan Lun has 398 sections with 113 herbal prescriptions, organised into the Six Divisions corresponding to the six stages of disease. Zhang Zhongjing, also called Zhang Ji developed the theory of external diseases, which arose when the body is out-of-balance. The development of traditional Chinese medicine (TCM) is similar to the theories of Galen. TCM holds that the body's vital energy (qi) circulates through channels, called meridians that have branches connected to bodily organs and functions. Concepts of the body and of disease used in TCM have notions similar to European humeral theory.

Proper organ function was also very important to Galen's views on anatomy and physiology. He tended to view health as the balanced, harmonious, optimal functioning of all the organs and systems of the body.

Both the Chinese and the Greco-Roman authors developed an objective, non-metaphysical medicine based on observation of patients and their response to treatment. Both these centres of culture as well as the Persian schools and Ayurveda removed superstition from the practice of medicine and created a rational approach to treatment. This created protocols and is based on empirical evidence. Empirical evidence is based on, concerned with, or verifiable by observation or experience. In the empiricist view, one can claim to have knowledge only when one has a true belief based on empirical evidence. This stands in contrast to the rationalist view under which reason or reflection alone is considered to be evidence for the truth or falsity of some propositions.

Today, when we read the works of these ancient masters, we see how naïve their view of disease was compared to our modern biomedical model. They did not, nor could not understand germ theory; they could not understand the complexity of the immune, endocrine or the nervous systems. Never the less, their empirical observations greatly improved medical practice and through their writings were able to profoundly codify treatments. Their approach lasted until the next development, scientific medicine.

\section{Limits to empiricism}

Medical observations are only guided by the skill of the individual practitioner, there is no external monitoring of process. Everything is dependent on 'expert opinion' and there are no objective processes to test the assumption or claims made by these experts. There was no pharmacology. 


\section{Scientific medicine}

The terms scientific medicine, allopathic medicine, biomedicine, conventional medicine, orthodox medicine, and Western medicine all refer to what in English is usually simply called medicine. Scientific medicine developed from but goes beyond empiricism. One of the breakthroughs was the germ theory, which was proposed in the mid$16^{\text {th }}$ century and gained widespread credence when substantiated by scientific discoveries of the $17^{\text {th }}$ through the late $19^{\text {th }}$ century. It supplanted earlier explanations for disease, such as Galen's humeral theory or the TCM theory of qi. Contemporary medicine applies biomedical sciences, biomedical research, genetics and medical technology to diagnose, treat, and prevent injury and disease, typically through pharmaceuticals or surgery. Scientific medicine, also called evidence-based medicine (EBM) is highly developed in the Western world due to the creation of the scientific method.

The scientific method is an ongoing process, which usually begins with observations about the natural world and is a body of techniques for investigating phenomena, acquiring new knowledge, or correcting and integrating previous knowledge. To be termed scientific, a method of inquiry is commonly based on empirical or measurable evidence subject to specific principles of reasoning. The Oxford English Dictionary defines the scientific method as "a method or procedure that has characterised natural science since the $17^{\text {th }}$ century, consisting in systematic observation, measurement, and experiment, and the formulation, testing, and modification of hypotheses.

A hypothesis is a conjecture, based on knowledge obtained while formulating the question. The hypothesis might be very specific or it might be broad. Scientists then test hypotheses by conducting experiments. The overall process of the scientific method involves making conjectures (hypotheses), deriving predictions from them as logical consequences, and then carrying out experiments based on those predictions. The scientific method is a logical and rational order of steps by which scientists come to conclusions about the world around them. The scientific method helps to organise thoughts and procedures so that scientists can be confident in the answers they find. Scientists use observations, hypotheses, and deductions to make these conclusions. In essence, it is about logical reasoning, testing to achieve on the best possible answer to a problem at any given time.

The scientific method has four steps:

1. Observation and description of a phenomenon or group of phenomena.

2. Formulation of a hypothesis to explain the phenomena. In physics, the hypothesis often takes the form of a causal mechanism or a mathematical relation.

3. Use of the hypothesis to predict the existence of other phenomena, or to predict quantitatively the results of new observations.

4. Performance of experimental tests of the predictions by several independent experimenters and properly performed experiments.

\section{Limitations of the scientific method}

Clearly, the scientific method is a powerful tool, but it does have its limitations. These limitations are based on the fact that a hypothesis must be testable and falsifiable and that experiments and observations be repeatable. This places certain topics beyond the reach of the scientific method. Science cannot prove or refute the existence of God or any other supernatural entity.

Personal and cultural beliefs also influence both perceptions and interpretations of natural phenomena. If the hypothesis-testing process fails to eliminate most of the personal and cultural biases, false hypotheses can survive the testing process and then be accepted as correct descriptions of the way the world works. The danger here is what is called 'Scientism'. Scientism is the dogmatic endorsement of scientific methodology and the reduction of all knowledge to only that which is measurable, it is a bias not readily acknowledged in medical science. Philosopher Tom Sorell provides this definition of scientism: "Scientism is a matter of putting too high a value on natural science in comparison with other branches of learning or culture." It has been defined as "the view that the characteristic inductive methods of the natural sciences are the only source of genuine factual knowledge and, in particular, that they alone can yield true knowledge about man and society."

In the 'pure' or hard sciences like physics and mathematics personal beliefs or perspectives hardly matter but in the 'softer' sciences like medicine, psychology or sociology there are always several perspectives on what is 'reality'. Robert Pearl, M.D. said, "doctors on the far "art" side of the spectrum maintain that every patient and physician is different. Therefore, they believe there is no one right way to treat a patient - labelling adherence to a common solution as "cookbook medicine." He concluded that "when two doctors approach the same problem in different ways, it's simply a matter of each doctor finding their own best solution for the patient's needs. They believe personal judgment and doctor's intuition are keys to highly personalised care".

Medicine is in essence, both a science and an art; it is scientific because we can ascertain causes of disease and their treatments objectively, one can determine the pharmaceutical activities of ant particular medicine. It is an art because each patient is unique and the expression of their disease, the polymorphism is particular to them and treatments are specific to the individual's needs. Disease is objective but the patient's needs and capacities are individual.

\section{Top down and bottom up science}

The scientific advances of the last several hundred years were due to a constellation of factors in the West. In a very few decades western culture leapt ahead of other cultures because of the rigour of science and the discipline of engineering. The approach was simple, reductionism and is derived from the work of people like Isaac Newton and René Descartes. In the sciences, application of methodological reductionism attempts explanation of entire systems in terms of their individual, constituent parts and their interactions. This is the top down approach. In a very simplified and sometimes contested form, reductionism is said to imply that a system is nothing but the sum of its parts, which of course simplifies the task of discovering what is seemingly true and what is not. Reductionism strongly reflects a certain perspective on causality that allows laser-like focus on specific phenomena. The scientific method only acknowledges monophasic consciousness. Tara W. Lumpkin $\mathrm{PhD}$, an environmental and medical anthropologist states, "The [reductionist] method is a specialised system that focuses on studying small and distinctive parts in isolation, which results in fragmented knowledge". This leads some people argue that inappropriate use of reductionism limits our understanding of complex systems and there is nothing more complex than a living, relating human being. 
The other approach to scientific enquiry, which is now emerging is called holism. Holism is a form of antireductionism, which is the complement of reductionism. This bottom up approach to science is the idea that natural systems (physical, biological, chemical, social, economic, mental, linguistic, medical) and their properties should be viewed as wholes, not as collections of parts. This often includes the view that systems function as wholes and that their functioning cannot be fully understood solely in terms of their component parts. In the latter half of the $20^{\text {th }}$ century, holism led to what is called Systems thinking. Systems in biology, psychology, medicine or sociology are frequently so complex that their behaviour is, or appears, "new" or "emergent": it cannot be deduced from the properties of the elements alone, one cannot predict the future from past events. Systems thinking led to Systems Biology, which is a biology-based inter-disciplinary field of study that focuses on complex interactions within biological systems, using a holistic approach to biological and biomedical research. Particularly from year 2000 onwards, the concept has been used widely in the biosciences in a variety of contexts. Systems Biology is about putting together rather than taking apart, integration rather than reduction. It requires that we develop ways of thinking about integration that are as rigorous as our reductionist programmes, but different. It means changing our philosophy, in the full sense of the term.

One of the outreaching aims of Systems Biology is to model and discover emergent properties, properties of cells, tissues and organisms functioning as a system whose theoretical description is only possible using techniques which fall under the remit of Systems Biology.

\section{Integrative medicine}

The future of medicine must and will be an integration of all the proceeding forms of medicine and a step into uncertainty. It must be scientific but the discipline of science must be reconfigured to include holism and reductionism as well as spirituality, psychophysical disciplines and biosocial networks. Until now medical science may have been the only remaining Newtonian science and needs deconstructing.

Deconstruction is part of a school of philosophy called Postmodernism, a late $20^{\text {th }}$ century movement based upon scepticism of 'modernism'. Modernism can be characterised by the belief in the existence of truth, objectivity, determinacy, causality and impartial observation. Modernism seeks to capture, define, understand and control knowledge, to find an absolute truth. Postmodernism, including deconstruction, questions the idea of certainty, the idea that an ultimate truth can be told, that there is an ultimate truth. This philosophical movement can be associated with the significant insights made in physics such as Einstein's 'Theory of Relativity' and Heisenberg's Quantum Physics. These 'discoveries' destroyed Newtonian physics and brought us into an age of uncertainty.
When one deconstructs a discipline one embraces a lack of control but a need to cooperate with others to find possible solutions through cooperation and communication. This is integrative medicine. Here is an editorial piece in the British Medical Journal titled Medicine, postmodernism, and the end of certainty (Dec 1996) by Paul Hodgkin. He said, "I came across a curious word the other day - Credicide; the death of belief. Not this or that one but all and every. Strictly speaking, of course, it means the active killing of belief rather than just its simple demise. What is dying of course is not just Progress, Education, Science, Justice, or God-though all these do look anaemic shadows of their former selves. What is dying is the House of Belief itself".

\section{The future of medicine}

The future of medicine is in the synthesis of all proceeding stages in medicine, gleanings of the best qualities from each epoch. Primitive medicine was communal medicine, everyone was engaged and medicine, and public health had a common root. Primitive medicine had a high spiritual component, essential to engaging the whole person. The empirical medicine of the second stage brought forward a more complex cosmology, integrating self, society and culture. This medicine made sense to people; it was linked to their everyday existence. Scientific medicine is at its core concerned with evidence and that needs preserving in the medicine of the future but the concept of evidence needs re-visioning, re-conceptualising.

All of this will make us all feel insecure but isn't that what happens when we embrace a major paradigm shift? The future of medicine lies in not knowing, of being innocent, of being naked. The future lies not in finding ultimate truth but in working creatively as if every patient were a new opportunity to rediscover the origins of medicine, the sacred, the emotional, the social and cultural aspects of the individual. To bring together the power of reductionism with the compassion of Holism means we see the patient not the disease.

Integrating the past into the future requires one to take the best of our medical ancestors and creating a dialogue amongst all types of practitioners as well as patients.

\section{Acknowledgments}

None.

\section{Conflict of interest}

The authors declare that there is no conflict of interest.

\section{Funding}

None. 